\title{
Long-term outcomes of percutaneous coronary intervention in grafts and native vessels in coronary artery bypass grafting patients with diabetes mellitus
}

\author{
Dong Liu", Xiao Cui", Xiaoliang Luo, Zhongwei Sun, Bo Xu, Shubin Qiao, Jiansong Yuan \\ Department of Cardiology, Fuwai Hospital, National Center for Cardiovascular Diseases, Chinese Academy of Medical Sciences and Peking Union \\ Medical College, Beijing 100037, China \\ Contributions: (I) Conception and design: J Yuan, S Qiao; (II) Administrative support: None; (III) Provision of study materials or patients: None; (IV) \\ Collection and assembly of data: D Liu, X Cui, X Luo, Z Sun, B Xu; (V) Data analysis and interpretation: D Liu, X Cui; (VI) Manuscript writing: All \\ authors; (VII) Final approval of manuscript: All authors. \\ \#These authors contributed equally to this work. \\ Correspondence to: Jiansong Yuan, MD, PhD; Shubin Qiao, MD, PhD. Department of Cardiology, Fuwai Hospital, National Center for Cardiovascular \\ Diseases, Chinese Academy of Medical Sciences and Peking Union Medical College, 167 North Lishi Road, Xicheng District, Beijing 100037, \\ China. Email: yuanjiansong@fuwaihospital.org; qiaoshubin@fuwaihospital.org.
}

Background: Atherosclerosis in diabetic patients progresses fast. Evidence on how to choose target vessels of percutaneous coronary interventions (PCIs) in diabetic patients post-coronary artery bypass graft (postCABG) is insufficient.

Methods: One hundred and fifty-seven patients with diabetes and previous CABG, who underwent PCI of either a graft vessel $(\mathrm{GV})(\mathrm{n}=44)$ or a native vessel $(\mathrm{NV})(\mathrm{n}=113)$ in the National Center for Cardiovascular Disease, China, were studied. In-hospital and long-term clinical outcomes were compared between the groups.

Results: Diabetic patients with prior CABG had more PCI to native arteries, but the proportion of grafts PCI increased as time went on. Both groups had similar baseline characteristics. Group GV patients compared with group NV had more totally occluded NVs, less totally occluded grafts and more in-stent restenosis. However, there was no difference in in-hospital mortality and long-term incidence of major adverse cardiac event (MACE), cardiac death, nonfatal myocardial infarction (MI), or revascularization. Multivariate logistic regression analysis showed that PCI success [hazard ratio (HR), 11.488; 95\% confidence interval (CI), 1.135-116.303; $\mathrm{P}<0.05]$ was independent predictor of MACE.

Conclusions: It suggested similar long-term clinical outcomes after PCI in GV or NV in prior CABG patients with diabetes. Thus, the vessel with higher estimated PCI success rate should be prioritized by operators.

Keywords: Stent; coronary artery bypass graft (CABG); percutaneous coronary intervention (PCI); diabetic mellitus

Submitted May 29, 2019. Accepted for publication Sep 27, 2019.

doi: $10.21037 /$ jtd.2019.10.33

View this article at: http://dx.doi.org/10.21037/jtd.2019.10.33

\section{Introduction}

Diabetes mellitus is a metabolic disease globally, with nearly 285 million adults affected worldwide (1). It is quite common that diabetic patients are complicated by coronary artery disease (CAD). Furthermore, atherosclerosis within diabetic patients is frequently diffuse and rapidly progressive $(2,3)$. Several large trials (4-8) and guidelines $(9,10)$ suggested that coronary artery bypass graft (CABG) 
surgery was prior to percutaneous coronary intervention (PCI) in diabetic patients with multi-vessel CAD. However, repeat revascularization is often required due to bypass graft failure, and lesion progression in the native vessels (NVs) $(11,12)$. Since redo-CABG has poorer clinical prognosis, $\mathrm{PCI}$ is the most common revascularization procedure after CABG (13). Nevertheless, information on choose either $\mathrm{NV}$ or graft vessel (GV) as the target of PCI is scarce, especially in diabetic patients. This research is to compare the long-term clinical outcome of diabetic patients with a history CABG following either NV or graft PCI.

\section{Methods}

This was an observational study, conducted in National Center for Cardiovascular Disease, China. We identified 171 consecutive diabetic patients with prior CABG, who subjected to PCI of either a graft or a NV from January 2009 to June 2015. Patients who underwent PCI in NVs and GVs simultaneously or missed follow-up data were excluded. No patients underwent staged PCI of NV and GV during the same admission. A total of 157 patients met the study criteria.

All patients were performed with standard PCI and treated with routine dual antiplatelet therapy (aspirin $300 \mathrm{mg}$ and clopidogrel $300 \mathrm{mg}$ or ticagrelor $180 \mathrm{mg}$ ) before PCI. Anticoagulation regimens were decided by operators and included unfractionated heparin. After PCI, aspirin $100 \mathrm{mg}$ per day indefinitely thereafter. Clopidogrel was prescribed for 12 months following drug-eluting stent.

Clinical follow-up information of the patients was obtained by review of clinic visit or by telephone. All patients provided written informed consent prior to undergoing urgent or elective PCI. The study complied with the Declaration of Helsinki and was approved by the Hospital's Institutional Ethical Review Board (Fuwai Hospital, National Center for Cardiovascular Diseases).

\section{Definitions}

Diabetes mellitus was defined as either a previous diagnosis of diabetes mellitus treated with diet, oral agents or insulin, or a new diagnosis during hospitalization upon the published guidelines.

Procedural success was defined as the residual diameter stenosis of $<50 \%$ in the absence of death, urgent repeat revascularization or new-onset myocardial infarction (MI) during hospitalization.
The primary end-point was major adverse cardiac events (MACEs), defined as the combined occurrence of cardiac death, nonfatal MI, or need for a new revascularization strategy [including target lesion revascularization (TLR) and target vessel revascularization (TVR)]. Nonfatal MI was defined as chest discomfort and/or ST-segment and/or T-wave abnormalities in at least 2 contiguous leads of the electrocardiogram and/or new pathologic $\mathrm{Q}$ waves or left bundle branch block accompanied by elevated troponin I or creatine kinase isoenzyme-MB (CK-MB). TLR was related to clinically driven repeat PCI or CABG of the previous stent or in the $5-\mathrm{mm}$ distal or proximal segments. TVR was defined as clinically driven repeat PCI or CABG related to the previously stented vessel.

\section{Statistical analysis}

Continuous normally distributed variables were reported as mean \pm standard deviation (SD) and categorical variables were presented as frequency (\%). The continuous variables between groups were compared with student $t$-test and categorical variables between groups were compared with chi-square test. Survival analysis was demonstrated by Kaplan-Meier curves and the curves were compared with the log-rank test. Multivariate adjusted Cox regression model was conducted to determine the risk factors associated with the MACEs among prior CABG patients who underwent PCI. The following variables were entered into the model: age, NV vs. GV intervention, durations of hospital stay and if PCI success or not. For all analyses reported, $\mathrm{P}$ values are 2 -sided, and $\mathrm{P}$ values $<0.05$ were considered significant. SAS version 9.3 (SAS Institute, Cary, NC, USA) was used to perform all the analyses.

\section{Results}

Between January $1^{\text {st }} 2009$ and June $1^{\text {st }} 2015,171$ consecutive diabetic patients with prior CABG were subjected to PCI in our hospital. Patients were excluded if PCI was performed in both GVs and NVs $(11 / 171 ; 6.4 \%)$ or if follow-up data were missing $(3 / 171 ; 1.8 \%)$. In the remaining 157 patients (mean age of $62.8 \pm 8.5 ; 76.4 \%$ men), most target vessels were native coronary arteries $(n=113,72.0 \%$, group NV) and less frequently GVs ( $\mathrm{n}=44,28.0 \%$, group GV). In group GV, 41 patients $(93.2 \%)$ were performed PCI in saphenous vein graft (SVG), 3 (6.8\%) in arterial graft, and nobody in both SVG and arterial graft. The percentage of grafts as PCI target vessels increased significantly after 5 years from 


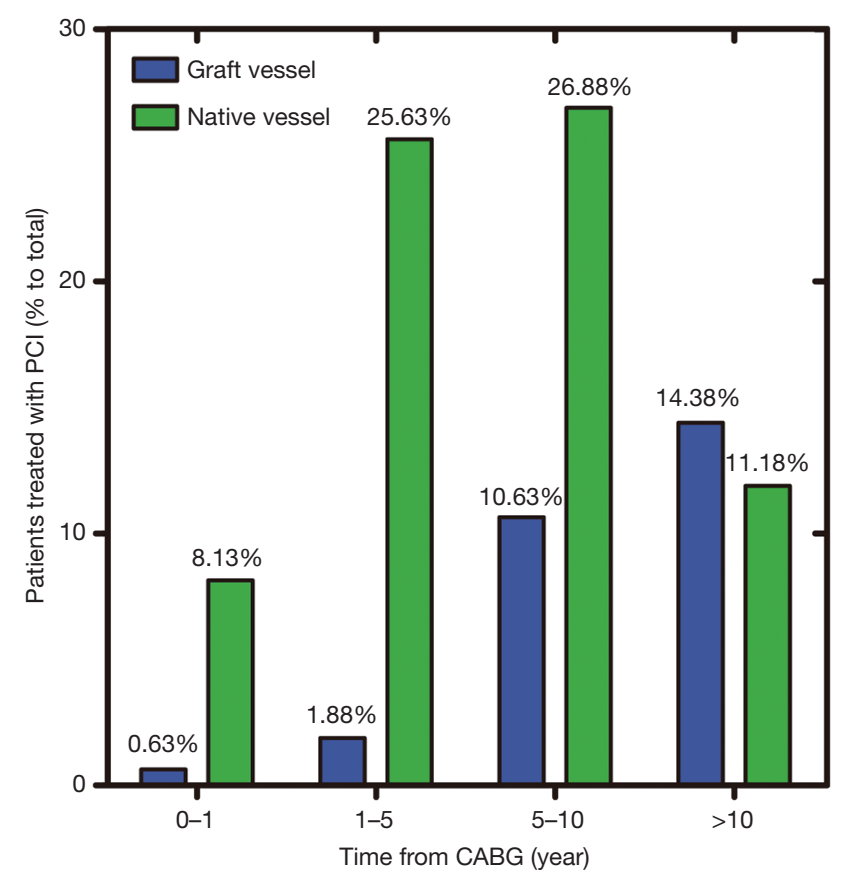

Figure 1 Comparison target vessel in diabetic patients with prior CABG. Comparison of the PCI target vessel in diabetic patients with prior $\mathrm{CABG}$ surgery with different time intervals from CABG. CABG, coronary artery bypass graft; PCI, percutaneous coronary intervention.

\section{CABG (Figure 1).}

There was no difference in most demographic characteristics and presentation symptom between the groups as depicted in Table 1. More group NV diabetic patients were combined with hypertension $(76.99 \%$ vs. $61.36 \%, \mathrm{P}=0.0486)$. Table 2 presented the baseline characteristics of the study population. Most stents implanted were drug eluting stents (DESs). The time from CABG to PCI was longer in group GV than group NV (118 vs. 71 months, $\mathrm{P}<0.001)$. Compared with group $\mathrm{NV}$, group GV patients had more occluded NVs $(\mathrm{P}=0.0049)$ and less occluded GVs $(\mathrm{P}<0.001)$. In group GV, 10 patients $(22.73 \%)$ were treated with distal protection device; none were used in $\mathrm{NV}(\mathrm{P}<0.001)$. Although group GV patients were treated with shorter stent length compared with group $\mathrm{NV}$ patients $(\mathrm{P}=0.0109)$, they presented more in-stent restenosis $(6.82 \%$ vs. $0.88 \%, \mathrm{P}=0.0341)$. Two groups of patients received similar drug treatment. Group GV patients had a lower pre-PCI thrombolysis in myocardial infarction (TIMI) flow grade compared with group NV ( $\mathrm{P}=0.0480)$. However, there was no significant difference in TIMI flow grade post-
PCI. Procedural success was achieved in 152 (96.82\%) patients $(97.73 \%$ in group GV and $96.46 \%$ in group $\mathrm{NV}$, $\mathrm{P}=0.6847)$.

The median follow-up of the research was $45 \pm 18$ months. There was no difference in the follow-up period after PCI performance in the two groups. None patients in two groups died during hospitalization. As for long-term outcomes (Table 3), 34 patients had MACEs (25.00\% vs. $20.35 \%$, $\mathrm{P}=0.5241)$ and 7 patients had cardiac death, 2 in group GV and 5 in the group $\mathrm{NV}(4.55 \%$ vs. $4.42 \%, \mathrm{P}=0.9738)$. There were 22 TVR (18.18\% vs. $12.39 \%, \mathrm{P}=0.3477), 17$ TLR ( $15.91 \%$ vs. $8.85 \%, \mathrm{P}=0.2524)$ and $8 \mathrm{MI}(6.82 \%$ vs. $4.42 \%$, $\mathrm{P}=0.6871$ ) without significant difference between the two groups. Kaplan-Meier plots showed that there was no difference in cumulative freedom from MACE $(64.4 \%$ vs. $74.1 \%, \mathrm{P}=0.479)$, cardiac death $(86.2 \%$ vs. $91.9 \%, \mathrm{P}=0.945)$, revascularization $(76.6 \%$ vs. $85.8 \%, \mathrm{P}=0.307)$ and nonfatal MI (92.6\% vs. 93.2\%, $\mathrm{P}=0.521)$ (Figure 2). Multivariate Cox regression analysis (Table 4) showed that PCI failure [hazard ratio (HR), 11.488; 95\% confidence interval (CI), $1.135-116.303 ; \mathrm{P}<0.05]$ was independently associated with MACE.

\section{Discussion}

Our research suggested that in prior CABG patients with diabetes mellitus, most PCIs (71.97\%) were performed in native coronary arteries, especially in the early stage. Previous studies focused mostly on general patients and showed different results: NV PCI was performed in $44 \%$ of 95 patients reported by Chen et al. (14) vs. $62.5 \%$ of 300,902 patients reported by Brilakis and colleagues (15). Chen et al. first showed that graft PCI accounted for the majority in the late stage (14), whereas Brilakis and colleagues suggested $\mathrm{NV}$ was the most common target vessel of PCI both early and late post-CABG (15). In a recent study, Brilakis et al. found that about $75 \%$ of PCIs involved native coronary artery lesions (16). However, the data from the group of diabetic patients was scarce. In 2002, Cole et al. found among diabetic patients, 43.19\% of PCI involved a SVG lesion (17). In our study, the most common target vessel of PCI in diabetic patients during early stage after CABG was native coronary artery. However, after 5 years, graft PCI increased significantly and even after 10 years, graft PCI accounted for more than half of PCI (Figure 1). One explanation for this finding may be lesions of SVG progress more slowly with longer 
Table 1 Baseline characteristics of the study participants

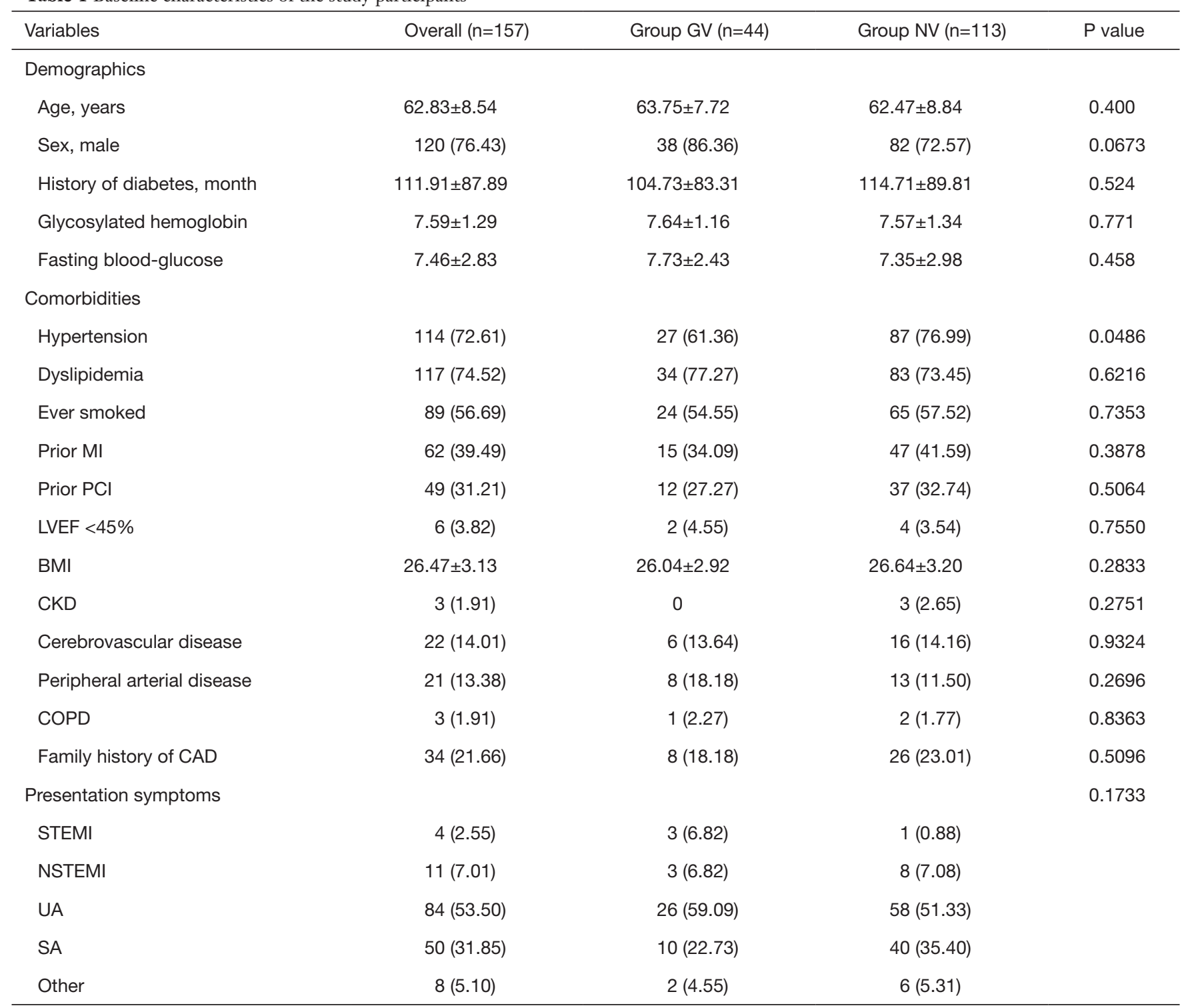

Data are presented as mean \pm SD or $n(\%)$. GV, graft vessel; NV, native vessel; MI, myocardial infarction; PCl, percutaneous coronary intervention; LVEF, left ventricular ejection fraction; BMI, body mass index; CKD, chronic kidney disease; COPD, chronic obstructive pulmonary disease; CAD, coronary artery disease; STEMI, ST-segment elevation myocardial infarction; NSTEMI, non-ST-segment elevation myocardial infarction; UA, unstable angina; SA, stable angina; SD, standard deviation.

interval from CABG than NV.

Moreover, we found that the choice of target vessel was associated with the severity of the lesions in NVs or GVs. NV PCI may be selected with diffusely degenerated grafts, whereas graft PCI may be preferred in the presence of severely occluded native coronary artery lesion. This conclusion is consistent with previous studies $(15,18)$.

Patients undergoing bypass graft interventions had higher in-stent restenosis (Table 2). Consistently, Keeley et al. (19) also reported SVG stenting restenosis rate was notably high. It is known that SVG interventions have higher risk for no-reflow $(20,21)$, whereas arterial grafts PCI can be complicated by long distance to the target lesion and increased tortuosity $(15,22)$, which probably renders this worse outcome.

The rate of distal embolic protection device use in our study was similar to the mean percentage in foreign hospitals $(22 \%$ among 19,546 SVG interventions 
Table 2 Procedure characteristics of study population

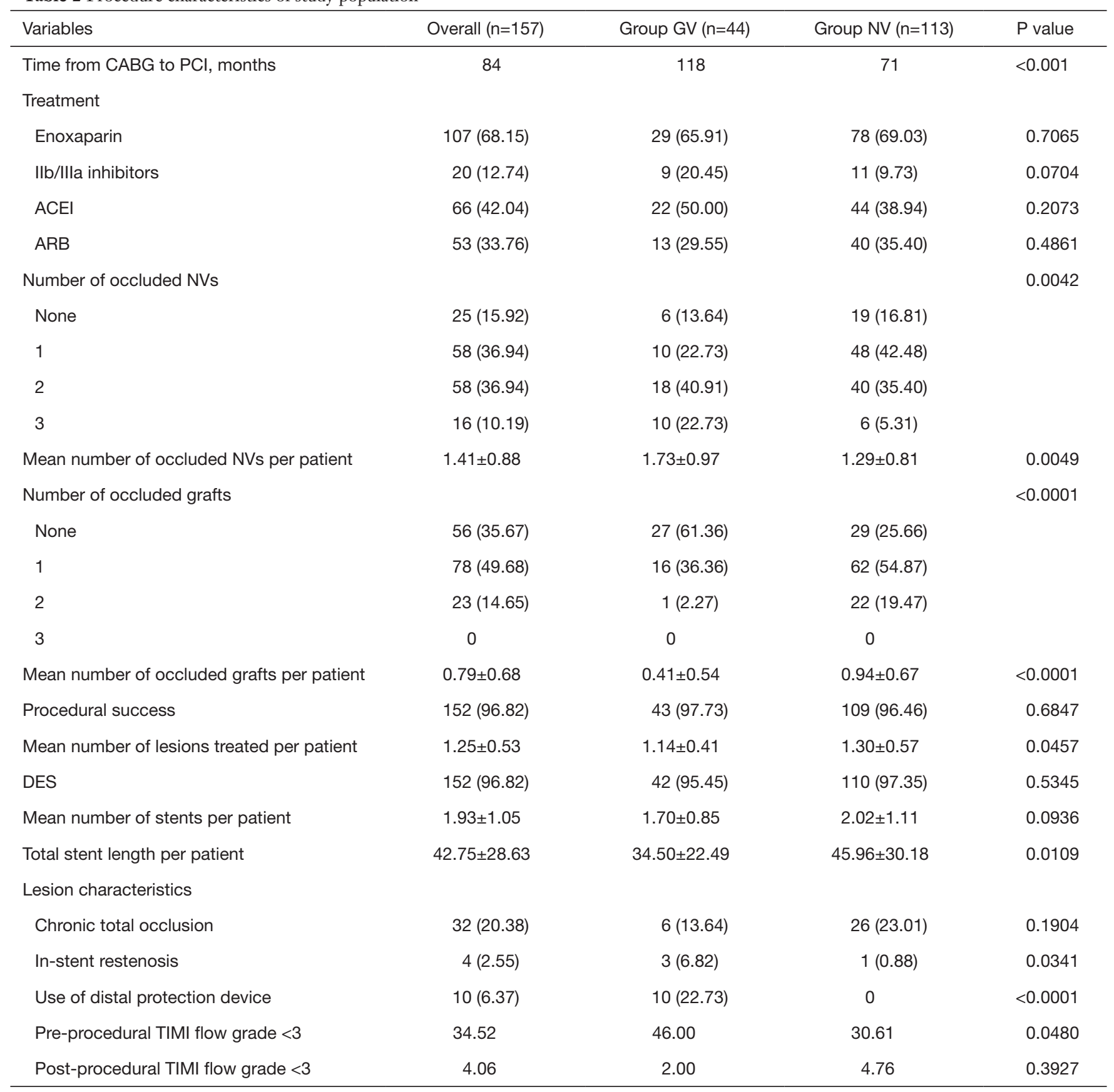

Data are presented as mean \pm SD or $\mathrm{n}(\%)$. GV, graft vessel; NV, native vessel; CABG, coronary artery bypass graft; PCl, percutaneous coronary intervention; ACEI, angiotensin-converting enzyme inhibitor; ARB, angiotensin receptor blocker; DES, drug eluting stent; TIMI, thrombolysis in myocardial infarction; SD, standard deviation.

included in the American College of Cardiology National Cardiovascular Data Registry) (23). With the start of proximal embolic protection devices, more and more SVG lesions can currently be protected (24). Thus, distal embolic protection in SVG interventions could potentially prevent procedural complications such as no-reflow and improve the procedural outcomes.

As for long-term clinical outcomes, we found that there 
Table 3 Outcomes of study population

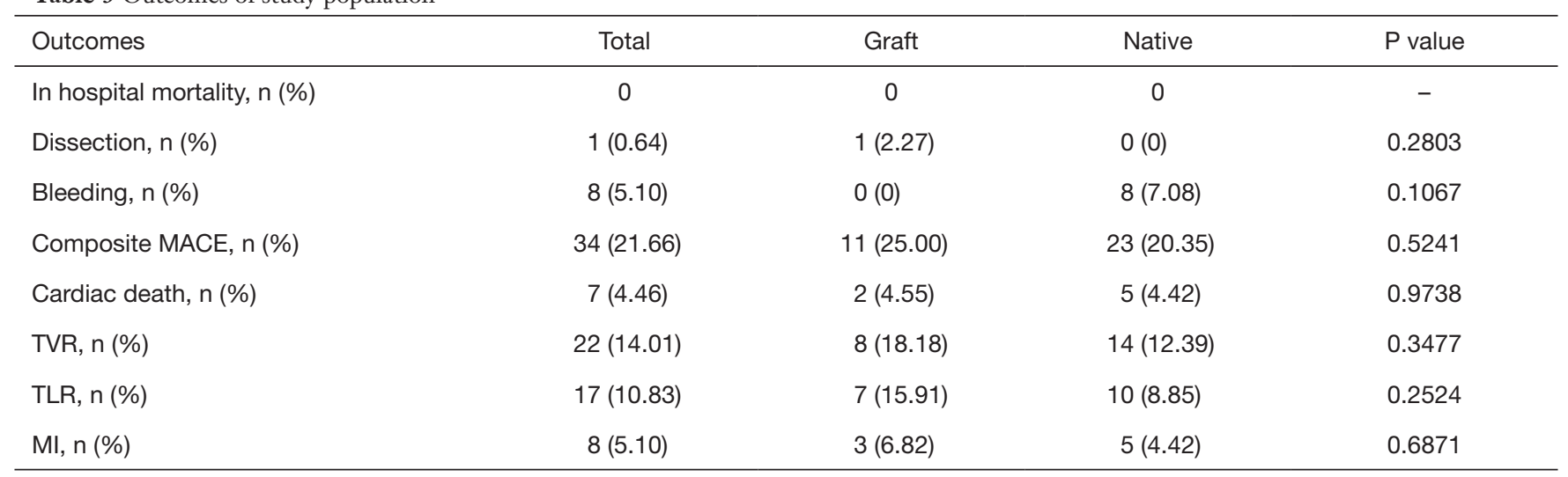

MACE, major adverse cardiac event; TVR, target vessel revascularization; TLR, target lesion revascularization, MI, myocardial infarction.
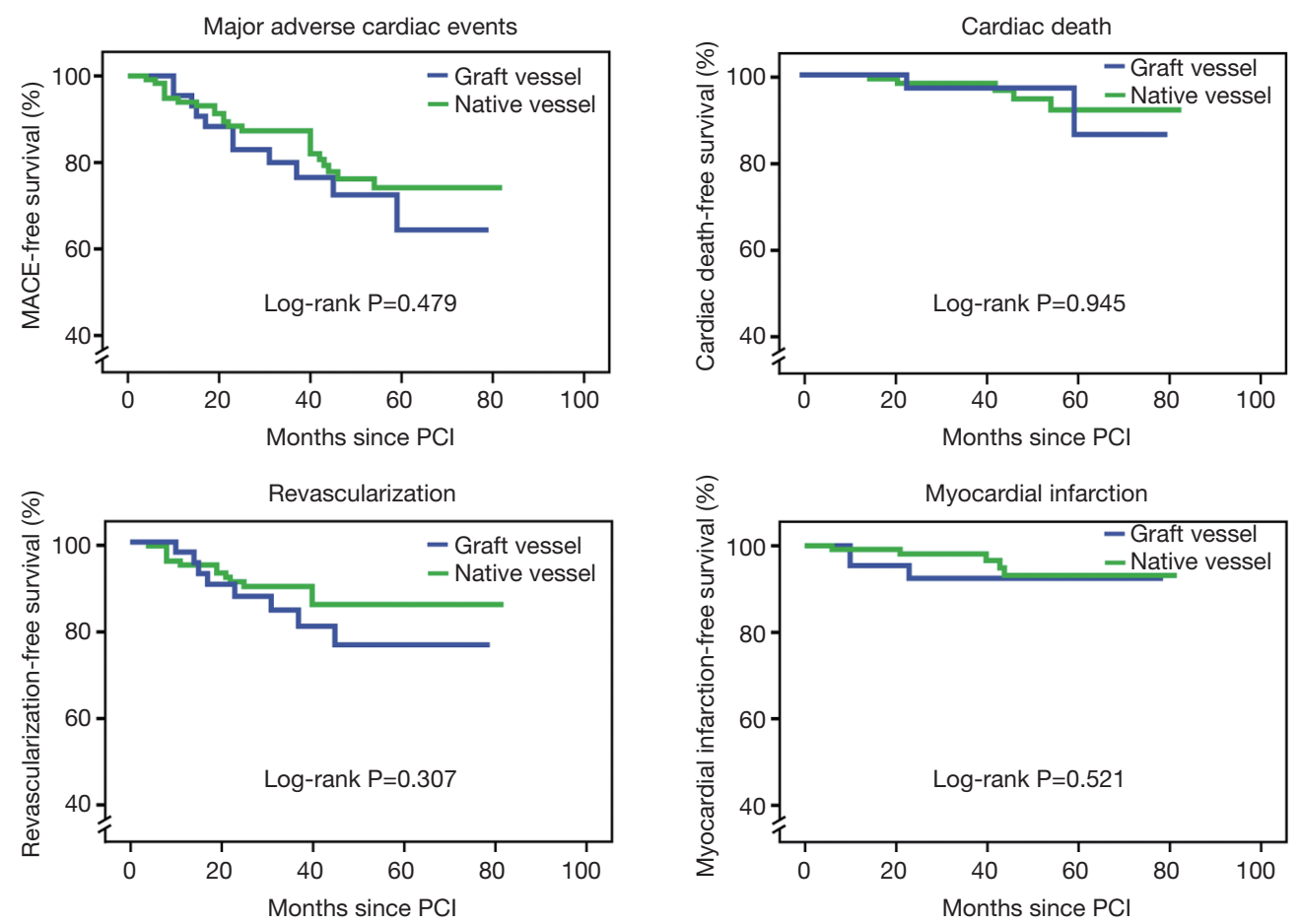

Figure 2 Kaplan-Meier curves for long-term clinical outcomes. Kaplan-Meier curves estimate event-free survival: freedom from MACEs; freedom from cardiac death, revascularization and nonfatal MI respectively. MACEs, major adverse cardiac events; MI, myocardial infarction; PCI, percutaneous coronary intervention.

was no difference in the incidence of MACE, cardiac death, MI, and revascularization in the two groups of diabetic patients. There were previous studies that compared clinical outcomes between general patients subjected to GV-PCI $v s$. NV-PCI, of which the results were inconclusive. Varghese et al. (18) made similar conclusions with us that both groups of patients had similar but relatively high incidence of MI
(5\%), repeat revascularization (9\%), and mortality $(6 \%)$ in a retrospective study of 142 patients with a mean follow-up of 2.5 years. Tejada et al. (25) also reported no difference in the incidence of MACEs, TLR, and death between two groups, but their study involved a smaller sample of 84 consecutive patients. Meliga and colleagues (26) presented a small series of 24 patients with chronic totally occluded SVG and found 
Table 4 Predictors of MACEs in patients with prior coronary bypass graft who underwent PCI

\begin{tabular}{lccc}
\hline Factors & P value & $\mathrm{HR}$ & $95 \% \mathrm{Cl}$ \\
\hline Age & 0.5382 & 0.985 & $0.940-1.033$ \\
Native vs. graft PCl & 0.3997 & 0.691 & $0.292-1.633$ \\
Time of hospital stay & 0.1182 & 1.079 & $0.981-1.187$ \\
PCl failure & 0.0387 & 11.488 & $1.135-116.303$ \\
\hline
\end{tabular}

MACE, major adverse cardiac event; HR, hazard ratio; Cl, confidence interval; PCl, percutaneous intervention.

no difference in the cumulative incidence of MACEs at 3 years between the two groups.

Interestingly, Bundhoo et al. (27) in a multi-center retrospective study of 161 post-CABG patients with a mean follow-up of 13.5 months, reported higher incidence rates of TVR $(15.0 \%$ vs. $4.9 \%, \mathrm{P}=0.03)$ and MACEs $(21.6 \%$ vs. $8.9 \%, \mathrm{P}=0.048)$ in the $\mathrm{GV}$ group. In the assessment of pexelizumab in acute myocardial infarction (APEXMI) trial, researchers found that bypass graft PCI patients had higher 90 -day mortality (19.0\% vs. $5.7 \%)$ compared with native artery PCI patient in a cohort of ST-segment elevation myocardial infarction (STEMI) patients with prior CABG undergoing PCI of the infarct-related artery (28). Similar conclusions were drawn in a research of 220 prior CABG patients from Mavroudis et al. (29) and in a pooledanalysis of 169 prior CABG patients with STEMI (30).

PCI to a native coronary artery has traditionally been recommended in prior CABG patients, because SVG PCI carries higher procedural risk and higher restenosis rates compared to native coronary PCI $(31,32)$. Current data was based on the general patients; however, the data focused on diabetic patients was scarce. Our study merely enrolled the group of diabetic patients and our outcome did not conflict with these recommendations, showing higher incidence of in-stent restenosis after SVG PCI than native coronary PCI. Unexpectedly, no significant difference in long-term clinical outcomes was seen between the native coronary and bypass graft PCI in diabetic patients. One potential explanation is the significantly diffuse and severe lesions in native coronary arteries of diabetic patient (33), which may increase difficulty in operation and subsequently offset the original benefit. Our multivariate analysis also revealed that PCI failure was independent predictor of MACE. From this point of view, it is reasonable that interventionalists should select the vessels easy to reach procedural success as targets. If both feasible, native coronary arteries could be the preferred target to prevent in-stent restenosis in diabetic patients (18,34,35). Besides, special focus should be placed on modifying aggressive risk factor to step down the disease progression $(36,37)$.

\section{Conclusions}

In our study, we found diabetic patients with prior CABG were liable to be performed with native arteries PCI. However, graft PCI become more preferred after longer time intervals from CABG. Higher rate of in-stent restenosis was seen in graft PCI. During hospitalization and after a 45-month follow-up, we found a similar mortality and incidence of composite MACE in both groups. PCI success or not was the predictor of MACE, which suggested that the vessel with higher estimated operating success rate should be selected as target.

\section{Acknowledgments}

Funding: This work was supported by the CAMS Innovation Fund for Medical Sciences (No. 2017-I2M-2-005 and 2016I2M-1-009).

\section{Footnote}

Conflicts of Interest: The authors have no conflicts of interest to declare.

Ethical Statement: The authors are accountable for all aspects of the work in ensuring that questions related to the accuracy or integrity of any part of the work are appropriately investigated and resolved. All patients provided written informed consent prior to undergoing urgent or elective PCI. The study complied with the Declaration of Helsinki and was approved by the Hospital's Institutional Ethical Review Board (Fuwai Hospital, National Center for Cardiovascular Diseases) (No. 2018-1034). 


\section{References}

1. Shaw JE, Sicree RA, Zimmet PZ. Global estimates of the prevalence of diabetes for 2010 and 2030. Diabetes Res Clin Pract 2010;87:4-14.

2. Arnett DK, Goodman RA, Halperin JL, et al. AHA/ ACC/HHS strategies to enhance application of clinical practice guidelines in patients with cardiovascular disease and comorbid conditions: from the American Heart Association, American College of Cardiology, and U.S. Department of Health and Human Services. J Am Coll Cardiol 2014;64:1851-6.

3. Huxley R, Barzi F, Woodward M. Excess risk of fatal coronary heart disease associated with diabetes in men and women: meta-analysis of 37 prospective cohort studies. BMJ 2006;332:73-8.

4. Huynh T, Perron S, O'Loughlin J, et al. Comparison of primary percutaneous coronary intervention and fibrinolytic therapy in ST-segment-elevation myocardial infarction: bayesian hierarchical meta-analyses of randomized controlled trials and observational studies. Circulation 2009;119:3101-9.

5. Serruys PW, Morice MC, Kappetein AP, et al. Percutaneous coronary intervention versus coronary-artery bypass grafting for severe coronary artery disease. N Engl J Med 2009;360:961-72.

6. Head SJ, Davierwala PM, Serruys PW, et al. Coronary artery bypass grafting vs. percutaneous coronary intervention for patients with three-vessel disease: final five-year follow-up of the SYNTAX trial. Eur Heart J 2014;35:2821-30.

7. Farkouh ME, Dangas G, Leon MB, et al. Design of the future revascularization evaluation in patients with diabetes mellitus: optimal management of multivessel disease (FREEDOM) trial. Am Heart J 2008;155:215-23.

8. Serruys PW, Unger F, Sousa JE, et al. Comparison of coronary-artery bypass surgery and stenting for the treatment of multivessel disease. N Engl J Med 2001;344:1117-24.

9. Fihn SD, Blankenship JC, Alexander KP, et al. 2014 ACC/AHA/AATS/PCNA/SCAI/STS focused update of the guideline for the diagnosis and management of patients with stable ischemic heart disease: a report of the American College of Cardiology/American Heart Association Task Force on Practice Guidelines, and the American Association for Thoracic Surgery, Preventive Cardiovascular Nurses Association, Society for Cardiovascular Angiography and Interventions, and Society of Thoracic Surgeons. J Thorac Cardiovasc Surg 2015;149:e5-23.

10. StephanWindecker, Kolh P, Alfonso F, et al. 2014 ESC/ EACTS guidelines on myocardial revascularization. Rev Esp Cardiol (Engl Ed) 2015;68:144.

11. Bourassa MG, Enjalbert M, Campeau L, et al. Progression of atherosclerosis in coronary arteries and bypass grafts: ten years later. Am J Cardiol 1984;53:102C-7C.

12. Hwang MH, Meadows WR, Palac RT, et al. Progression of native coronary artery disease at 10 years: insights from a randomized study of medical versus surgical therapy for angina. J Am Coll Cardiol 1990;16:1066-70.

13. Morrison DA, Sethi G, Sacks J, et al. Percutaneous coronary intervention versus repeat bypass surgery for patients with medically refractory myocardial ischemia: AWESOME randomized trial and registry experience with post-CABG patients. J Am Coll Cardiol 2002;40:1951-4.

14. Chen L, Théroux P, Lespérance J, et al. Angiographic features of vein grafts versus ungrafted coronary arteries in patients with unstable angina and previous bypass surgery. J Am Coll Cardiol 1996;28:1493-9.

15. Brilakis ES, Rao SV, Banerjee S, et al. Percutaneous coronary intervention in native arteries versus bypass grafts in prior coronary artery bypass grafting patients: a report from the National Cardiovascular Data Registry. JACC Cardiovasc Interv 2011;4:844-50.

16. Brilakis ES, O'Donnell CI, Penny W, et al. Percutaneous coronary intervention in native coronary arteries versus bypass grafts in patients with prior coronary artery bypass graft surgery: insights from the veterans affairs clinical assessment, reporting, and tracking program. JACC Cardiovasc Interv 2016;9:884-93.

17. Cole JH, Jones EL, Craver JM, et al. Outcomes of repeat revascularization in diabetic patients with prior coronary surgery. J Am Coll Cardiol 2002;40:1968-75.

18. Varghese I, Samuel J, Banerjee S, et al. Comparison of percutaneous coronary intervention in native coronary arteries vs. bypass grafts in patients with prior coronary artery bypass graft surgery. Cardiovasc Revasc Med 2009;10:103-9.

19. Keeley EC, Velez CA, O'Neill WW, et al. Long-term clinical outcome and predictors of major adverse cardiac events after percutaneous interventions on saphenous vein grafts. J Am Coll Cardiol 2001;38:659-65.

20. Banerjee S, Brilakis ES. Embolic protection during saphenous vein graft interventions. J Invasive Cardiol 2009;21:415-7.

21. Brilakis ES, Saeed B, Banerjee S. Drug-eluting stents in saphenous vein graft interventions: a systematic review. 
EuroIntervention 2010;5:722-30.

22. Lichtenwalter C, Banerjee S, Brilakis ES. Dual guide catheter technique for treating native coronary artery lesions through tortuous internal mammary grafts: separating equipment delivery from target lesion visualization. J Invasive Cardiol 2010;22:E78-81.

23. Mehta SK, Frutkin AD, Milford-Beland S, et al. Utilization of distal embolic protection in saphenous vein graft interventions (an analysis of 19,546 patients in the American College of Cardiology-National Cardiovascular Data Registry). Am J Cardiol 2007;100:1114-8.

24. Mauri L, Cox D, Hermiller J, et al. The PROXIMAL trial: proximal protection during saphenous vein graft intervention using the Proxis Embolic Protection System: a randomized, prospective, multicenter clinical trial. J Am Coll Cardiol 2007;50:1442-9.

25. Tejada JG, Velazquez M, Hernandez F, et al. Percutaneous revascularization in patients with previous coronary artery bypass graft surgery. Immediate and 1-year clinical outcomes. Int J Cardiol 2009;134:201-6.

26. Meliga E, García-García HM, Kukreja N, et al. Chronic total occlusion treatment in post-CABG patients: saphenous vein graft versus native vessel recanalizationlong-term follow-up in the drug-eluting stent era. Catheter Cardiovasc Interv 2007;70:21-5.

27. Bundhoo SS, Kalla M, Anantharaman R, et al. Outcomes following PCI in patients with previous CABG: a multi centre experience. Catheter Cardiovasc Interv 2011;78:169-76.

28. Welsh RC, Granger CB, Westerhout CM, et al. Prior coronary artery bypass graft patients with ST-segment elevation myocardial infarction treated with primary percutaneous coronary intervention. JACC Cardiovasc Interv 2010;3:343-51.

29. Mavroudis CA, Kotecha T, Chehab O, et al. Superior long term outcome associated with native vessel versus graft vessel PCI following secondary PCI in patients with prior
CABG. Int J Cardiol 2017;228:563-9.

30. Nguyen TT, O'Neill WW, Grines CL, et al. One-year survival in patients with acute myocardial infarction and a saphenous vein graft culprit treated with primary angioplasty. Am J Cardiol 2003;91:1250-4.

31. Nguyen T, Pham L, Cheem TH, et al. Approach to the patient with prior bypass surgery. J Interv Cardiol 2004;17:339-46.

32. Kolh P, Windecker S, Alfonso F, et al. 2014 ESC/EACTS Guidelines on myocardial revascularization: the Task Force on Myocardial Revascularization of the European Society of Cardiology (ESC) and the European Association for Cardio-Thoracic Surgery (EACTS). Developed with the special contribution of the European Association of Percutaneous Cardiovascular Interventions (EAPCI). Eur J Cardiothorac Surg 2014;46:517-92.

33. Martinez SC, Holmes DR. Controversies surrounding percutaneous coronary intervention in the diabetic patient. Expert Rev Cardiovasc Ther 2016;14:633-48.

34. Ellis SG, Brener SJ, DeLuca S, et al. Late myocardial ischemic events after saphenous vein graft intervention-importance of initially "nonsignificant" vein graft lesions. Am J Cardiol 1997;79:1460-4.

35. Pendyala LK, Loh JP, Kitabata H, et al. The impact of diabetes mellitus on long-term clinical outcomes after percutaneous coronary saphenous vein graft interventions in the drug-eluting stent era. J Interv Cardiol 2014;27:391-8.

36. Xanthopoulou I, Davlouros P, Tsigkas G, et al. Long-term clinical outcome after percutaneous coronary intervention in grafts vs native vessels in patients with previous coronary artery bypass grafting. Can J Cardiol 2011;27:716-24.

37. Boatman DM, Saeed B, Varghese I, et al. Prior coronary artery bypass graft surgery patients undergoing diagnostic coronary angiography have multiple uncontrolled coronary artery disease risk factors and high risk for cardiovascular events. Heart Vessels 2009;24:241-6.
Cite this article as: Liu D, Cui X, Luo X, Sun Z, Xu B, Qiao $\mathrm{S}$, Yuan J. Long-term outcomes of percutaneous coronary intervention in grafts and native vessels in coronary artery bypass grafting patients with diabetes mellitus. J Thorac Dis 2019;11(11):4798-4806. doi: 10.21037/jtd.2019.10.33 